\title{
Transport actif et sécuritaire des élèves du primaire : analyse comparée des risques de blessures associés aux déplacements des enfants en automobile, à pied et à vélo entre la maison et l'école
}

\author{
M. Lavoie, M.D. (1); G. Burigusa, M.A., M. Sc. (1); P. Maurice, M.D., FRCPC (1); D. Hamel, M. Sc. (2); É. Turmel, B. Sc. (3)
}

Cet article a fait l'objet d'une évaluation par les pairs.

Diffuser cet article sur Twitter

\section{Résumé}

Introduction : Les programmes de promotion du transport actif au primaire visent à contrer la sédentarité chez les jeunes en provoquant un transfert modal des déplacements en automobile vers les déplacements à pied ou à vélo auprès des enfants demeurant à une distance de l'école propice à la marche ou au vélo. Cette étude a pour objectif d'évaluer le risque de blessures lié aux déplacements à pied, à vélo et en automobile entre la maison et l'école pour les élèves du primaire de la région de Montréal, ainsi que d'évaluer l'impact d'un transfert modal des déplacements en automobile vers les déplacements à pied ou à vélo sur le nombre d'enfants blessés dans cette région.

Méthodologie : Le risque de blessures a été estimé pour la période 2003 à 2007 en calculant le taux annuel moyen de blessés chez les 5 à 12 ans en tant que piétons, cyclistes ou occupants d'une automobile, par 100 millions de kilomètres parcourus lors des heures habituelles de déplacements entre la maison et l'école, durant la période scolaire. L'impact d'un transfert modal des déplacements en automobile a été évalué auprès des enfants demeurant à une distance de l'école propice à la marche et au vélo (moins de 1,6 km), soit la clientèle visée par les programmes de promotion du transport actif au primaire. Cette évaluation a été faite en utilisant le taux régional de blessures calculé pour chaque mode de déplacement.

Résultats : Entre 2003 et 2007, en moyenne, 168 enfants de 5 à 12 ans ont été blessés, chaque année, comme piétons (64), cyclistes (28) et occupants d'une automobile (76), dans la région de Montréal, lors des heures habituelles de déplacement entre la maison et l'école. Le taux de blessures a été de 69 blessés par 100 millions de kilomètres pour les déplacements en automobile (groupe de référence), 314 blessés pour les piétons (risque relatif : 4,6; IC à $95 \%$ : 4,3 à 5,1) et 1519 blessés pour les cyclistes (risque relatif : 22,2; IC à $95 \%: 14,3$ à 30,0). Un transfert de $20 \%$ du kilométrage parcouru en automobile par les enfants demeurant à moins de 1,6 km de l'école aurait occasionné, dans le cas d'un transfert vers la marche, 3,7 blessés de plus par année, soit une augmentation de 2,2 \% du nombre annuel moyen d'enfants blessés lors des heures habituelles de déplacement entre la maison et l'école dans la région de Montréal et, dans le cas d'un transfert vers le vélo, de 24,4 blessés, soit une augmentation de 14,5\%.

Conclusion : Le risque de blessures chez les élèves du primaire lors des heures habituelles de déplacement entre la maison et l'école est plus élevé pour les déplacements à pied et à vélo que pour les déplacements en automobile, et les enfants se déplaçant à vélo courent plus de risques de se blesser que ceux se déplaçant à pied. Un transfert modal des déplacements en automobile augmenterait le nombre d'enfants blessés à l'échelle de la région (blessures légères pour la plupart), si aucune mesure n'était prise pour réduire le risque de blessures chez les piétons et les cyclistes.

Mots-clés : transport actif, école primaire, blessures, piétons, cyclistes, déplacements, risques

\section{Introduction}

Au cours des dernières années, plusieurs initiatives ont vu le jour dans bon nombre de pays industrialisés afin de promouvoir le transport actif auprès des élèves du primaire. Parmi les plus connues, il y a le programme Safe Routes to School ${ }^{1}$. Au Québec, la promotion du transport actif auprès des élèves du primaire se concrétise principalement par la mise en œuvre du programme "Mon école à pied, à vélo » ${ }^{2}$. Dans l'ensemble, ces programmes ont pour principal objectif de diminuer la sédentarité chez les jeunes ainsi que les problèmes de santé qui y sont associés en provoquant un transfert des déplacements, entre la maison et l'école, en automobile vers les déplacements à pied ou à vélo. Ce type de programmes provoque habituellement un transfert de $20 \%$ ou moins des déplacements en automobile auprès de la clientèle ciblée ${ }^{3}$.

La sécurité routière est une dimension importante des programmes de promotion du transport actif auprès des élèves du primaire. Les jeunes de cet âge n'ont pas toujours les capacités cognitives et psychomotrices requises pour se déplacer à pied ou à vélo de façon sécuritaire ${ }^{4}$. De plus, l'insécurité routière est l'une des principales raisons invoquées par les parents pour privilégier l'automobile comme mode de déplacement de leurs enfants entre la maison et l'école ${ }^{5-7}$.

Une étude réalisée aux États-Unis a montré que les enfants de 5 à 13 ans se déplaçant à pied ou à vélo pour se rendre à

Rattachement des auteurs :

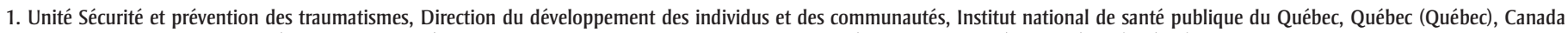
2. Direction de l'analyse et de l'évaluation des systèmes de soins et services, Institut national de santé publique du Québec, Québec (Québec), Canada

3. Direction des études et des stratégies en sécurité routière, Société de l'assurance automobile du Québec, Québec (Québec), Canada

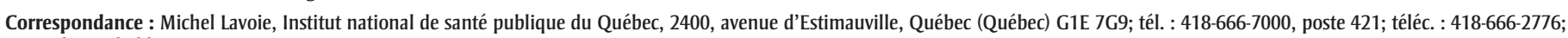
courriel : michel.lavoie@inspq.qc.ca 
l'école courent davantage de risques de blessures que ceux voyageant en automo$b e^{8}$. Une autre étude réalisée en Nouvelle-Zélande a montré les mêmes tendances ${ }^{9}$. Les résultats de ces études laissent présager que les programmes de promotion du transport actif pourraient avoir pour effet d'augmenter le nombre d'enfants blessés comme piétons ou cyclistes en raison d'un transfert modal, mais un tel effet n'a pas encore été documenté scientifiquement.

Cette étude a pour objectif d'évaluer, dans la région de Montréal, le risque de blessures auprès des élèves du primaire lors de leurs déplacements à pied, à vélo et en automobile, entre la maison et l'école. Cette étude a également pour objectif d'évaluer l'impact d'un transfert modal des déplacements en automobile vers les déplacements à pied ou à vélo entre la maison et l'école sur le nombre d'enfants blessés dans cette région. La région de Montréal revêt un intérêt particulier pour ce type d'évaluation, car elle est propice à la promotion des déplacements actifs en raison de son caractère urbain, elle regroupe environ la moitié des jeunes en âge de fréquenter l'école primaire au Québec et elle fait partie des régions pour lesquelles des données sur les déplacements des enfants entre la maison et l'école sont disponibles.

\section{Méthodologie}

La population à l'étude est constituée des enfants de 5 à 12 ans demeurant sur le territoire couvert par l'Enquête originedestination menée dans la région de Montréal en 2003 (c'était l'enquête de ce type la plus récente au moment où notre étude a été réalisée). Ce territoire couvre $5500 \mathrm{~km}^{2}$ et regroupe 88 municipalités, dont celles de Montréal, Longueuil et Laval. En 2003, il regroupait 52,3 \% de tous les enfants de 5 à 12 ans du Québec ${ }^{10}$.

\section{Risque de blessures}

Le risque de blessures a été estimé pour la période 2003-2007 en calculant le taux annuel moyen de blessés chez les enfants de 5 à 12 ans comme piétons, cyclistes ou occupants d'une automobile, par 100 millions de kilomètres parcourus lors des heures habituelles de déplacement entre la maison et l'école, durant la période scolaire. Ici, le terme automobile réfère au type de véhicule motorisé habituellement utilisé par les parents entre la maison et l'école, ce qui inclut, outre les automobiles, les camionnettes et les véhicules utilitaires sport (sont exclus les autres types de véhicules motorisés dont les autobus, les camions lourds, les véhicules commerciaux et les véhicules toutterrain).

\section{Source des données}

Les données sur les blessés (numérateur du taux) proviennent du Fichier des rapports d'accidents de véhicules routiers complétés par les policiers ${ }^{11}$. Ce fichier comprend des données sur tous les résidents du Québec ayant été blessés en tant que piétons, cyclistes ou occupants d'un véhicule routier lors d'une collision impliquant un véhicule motorisé en mouvement sur la voie publique. Les victimes sont classées selon la sévérité de leurs blessures, sur la base des données consignées par les policiers : blessures mortelles, graves ou légères. Les données sur le kilométrage parcouru selon le type de déplacement (dénominateur du taux) proviennent de l'Enquête origine-destination menée dans la région de Montréal en $2003^{12}$. Cette enquête s'est déroulée du 2 septembre au 20 décembre auprès d'un échantillon représentatif des ménages vivant sur le territoire de l'une ou l'autre des 88 municipalités du territoire couvert par l'enquête. Les ménages ont été échantillonnés aléatoirement au sein de l'ensemble des strates géographiques de ce territoire (et non pas municipalité par municipalité) durant toute la durée de l'enquête, afin d'assurer une bonne distribution de ces ménages selon les périodes de l'enquête et selon leur composition (ménage avec enfant ou non). Les données ont été recueillies durant un jour de la semaine excepté le lundi, au moyen d'une entrevue téléphonique réalisée auprès d'un adulte membre du ménage échantillonné. Le répondant a été questionné sur tous les déplacements effectués par chacun des membres de son ménage durant la journée précédant l'entrevue. Les données ainsi recueillies permettent notamment de connaître le mode de transport utilisé (ex. : marche, vélo, automobile) et la distance parcourue lors de chaque déplacement (correspond à la distance à vol d'oiseau, soit la longueur d'une ligne droite tracée entre le point de départ et le point d'arrivée de chaque déplacement).

\section{Numérateur du taux}

Le nombre d'enfants blessés a été déterminé en retenant les victimes âgées de 5 à 12 ans ayant été blessées comme piétonnes, cyclistes ou occupantes d'une automobile, lors des heures habituelles de déplacement des enfants entre la maison et l'école, durant la période scolaire. Les enfants ayant été blessés sur le territoire couvert par l'enquête ont été identifiés par le code municipal du lieu de survenue de l'incident à l'origine des blessures, qui est inclus dans le Fichier des rapports d'accidents de véhicules routiers. Les heures habituelles de déplacement des enfants entre la maison et l'école ont été définies suivant trois plages horaires : 7 h 00 à 8 h 59, 11 h 00 à 12 h 59 et 15 h 00 à 16 h 29. La dernière plage horaire se termine à 16 h 29 et non à 18 h 00 de manière à exclure les enfants ayant été blessés après leur retour à la maison (ex. : enfants blessés en jouant dans la rue, en face de chez eux). La période scolaire a été fixée à 200 jours par année, ce qui exclut les vacances d'été (24 juin au 31 août) et celles des Fêtes (24 décembre au 2 janvier) ainsi que tous les samedis et dimanches et les congés fériés locaux : le Vendredi Saint, le lundi de Pâques, la Fête du travail, l'Action de grâce et la Journée des Patriotes. Les enfants blessés comme occupants d'une automobile ont été identifiés en utilisant la catégorie blessés par automobile-camionnette du Fichier des rapports d'accidents, laquelle comprend les automobiles, les camions légers et les véhicules utilitaires sport.

\section{Dénominateur du taux}

Le nombre de kilomètres parcourus a été déterminé en estimant le nombre total de kilomètres parcourus en une année par l'ensemble des enfants de 5 à 12 ans habitant sur le territoire sélectionné pour l'enquête, lors des heures habituelles de déplacement entre la maison et l'école, durant la période scolaire. Cette estima- 
tion a été obtenue en calculant dans un premier temps le kilométrage parcouru en une journée, à pied, à vélo et en automobile par les enfants de 5 à 12 ans ayant participé à l'enquête (l'échantillon), lors des heures habituelles de déplacement entre la maison et l'école, durant la période scolaire. Les déplacements effectués en automobile ont été identifiés en utilisant la catégorie automobile-passager, laquelle comprend les enfants ayant circulé en automobile, en camionnette et en véhicule utilitaire sport (comme pour le numérateur). Dans certains cas, la distance parcourue lors d'un même déplacement représentait une valeur extrême. Un traitement a alors été appliqué afin de minimiser l'impact de ces valeurs extrêmes sur le nombre total de kilomètres parcourus : les déplacements à pied de plus de $4 \mathrm{~km}$ ont été ramenés à $4 \mathrm{~km}$, ceux à vélo de plus de $8 \mathrm{~km}$ ont été ramenés à $8 \mathrm{~km}$ et ceux en automobile de plus de $50 \mathrm{~km}$ ont été ramenés à $50 \mathrm{~km}$. Au total, 13 valeurs extrêmes ont été traitées pour les déplacements à pied, 14 pour les déplacements à vélo et 22 pour les déplacements en automobile, ce qui correspond respectivement à 0,15\%, 4,3\% et $0,26 \%$ du total des déplacements à pied, à vélo et en automobile effectués dans cette région en 2003. Dans un deuxième temps, parce que les enquêtes origine-destination fournissent des données sur les déplacements effectués pendant une seule journée dans la semaine, les données sur le kilométrage parcouru calculées pour l'échantillon ont été multipliées par 200, afin d'obtenir des valeurs se rapportant à une période de 200 jours (comme pour le numérateur). Enfin, un facteur de pondération d'enquête a été utilisé pour permettre d'inférer, à l'ensemble de la population des jeunes de 5 à 12 ans résidant sur le territoire couvert par l'enquête (la population), le nombre total de kilomètres parcourus estimé par l'échantillon.

\section{Risque relatif}

Le risque relatif de blessures lors des heures habituelles de déplacement des enfants entre la maison et l'école a été mesuré en comparant les taux de blessures liés aux déplacements à pied et à vélo par rapport au taux de blessures des occupants d'une automobile. Le calcul des erreurstypes des estimations pour le nombre de kilomètres parcourus tient compte des facteurs de pondération de même que des effets de plan dus à l'échantillonnage complexe des enquêtes origine-destination. Par exemple, les déplacements des enfants de 5 à 12 ans provenant d'un même ménage enquêté ou d'un même voisinage ne peuvent être considérés comme indépendants et forment en fait des grappes (clusters) d'observations. L'utilisation d'un logiciel spécialisé (SUDAAN) a permis, en spécifiant les paramètres du plan de sondage, de corriger les erreurs-types des estimations de populations et, par conséquent, des rapports de taux ${ }^{13}$. Il est ainsi possible de déterminer des intervalles de confiance avec un niveau de signification à $95 \%$, tant pour les taux de blessures que pour la mesure du risque relatif.

\section{Impact d'un transfert modal}

L'impact d'un transfert modal des déplacements en automobile vers les déplacements à pied ou à vélo sur le nombre d'enfants blessés entre la maison et l'école a été évalué à partir du taux de blessures lié à chacun de ces modes de déplacement. Cette évaluation a été faite en considérant uniquement les enfants se déplaçant en automobile entre la maison et l'école et demeurant à moins de 1,6 km de l'école (distance raisonnable de marche et de vélo), soit la clientèle ciblée par les programmes visant à promouvoir le transport actif auprès des élèves du primaire. En 2003, le nombre d'enfants de 5 à 12 ans respectant ces deux conditions était estimé à 68900 dans la région de Montréal (territoire couvert par l'enquête), ce qui représentait 57,5 \% de tous les enfants se déplaçant en automobile entre la maison et l'école dans cette région. En 2003, ces 68900 enfants ont parcouru $8815400 \mathrm{~km}$ en automobile lors des heures habituelles de déplacement entre la maison et l'école, durant la période scolaire. L'impact d'un transfert modal des déplacements en automobile sur le nombre d'enfants blessés a été évalué en postulant que $10 \%, 20 \%$, $30 \%, 40 \%$ et $50 \%$ du kilométrage parcouru par ces enfants se ferait à pied ou à vélo plutôt qu'en automobile. Cette évaluation a été faite en comparant le nombre d'enfants susceptibles de se blesser comme piétons, cyclistes ou occupants d'une automobile en parcourant le kilométrage associé à chacun de ces cinq scénarios de transfert (un transfert de $10 \%$ correspondant à 881540 kilomètres). Le nombre potentiel d'enfants blessés comme piétons, cyclistes ou occupants d'une automobile a été estimé à partir des taux de blessures calculés à l'échelle du territoire enquêté pour les enfants se déplaçant à pied, à vélo et en automobile durant la période 20032007 (taux régional de blessures). Cette estimation a été faite en supposant que le transfert modal des déplacements en automobile se ferait entièrement au profit soit des déplacements à pied, soit des déplacements à vélo. L'écart observé pour un scénario de transfert donné entre le nombre d'enfants blessés comme piétons et comme occupants d'une automobile a été exprimé en nombre de blessés additionnels et en pourcentage du nombre annuel moyen d'enfants blessés lors des heures habituelles de déplacement entre la maison et l'école. La même procédure a été répétée pour les scénarios impliquant un transfert des déplacements en automobile vers les déplacements à vélo.

\section{Résultats}

\section{Nombre d'enfants blessés}

Dans la région de Montréal, entre 2003 et 2007, 957 enfants âgés de 5 à 12 ans ont été blessés comme piétons, cyclistes ou occupants d'un véhicule motorisé (incluant les camions lourds et les autres types de véhicules routiers) en moyenne chaque année, ce qui représente 46,8 \% (957) 2 044) de l'ensemble des cas observés à l'échelle du Québec (figure 1). De ce nombre, 178 enfants (18,6\%) ont été blessés lors des heures habituelles de déplacement entre la maison et l'école, durant la période scolaire, dont 64 piétons, 28 cyclistes et 76 occupants d'une automobile, d'une camionnette ou d'un véhicule utilitaire sport (sont exclus les occupants d'un autre type de véhicule, dont les camions lourds et les autobus). Dans la plupart des cas, il s'agissait de blessures légères $(89,1 \%$ des piétons, 97,1 \% des cyclistes et 97,6\% des occupants d'une automobile, d'une camionnette ou d'un véhicule utilitaire sport [données non présentées]). 
FIGURE 1

Processus d'identification des enfants de 5 à 12 ans blessés lors des heures habituelles de déplacement entre la maison et l'école (nombre annuel moyen), région de Montréal, 2003-2007

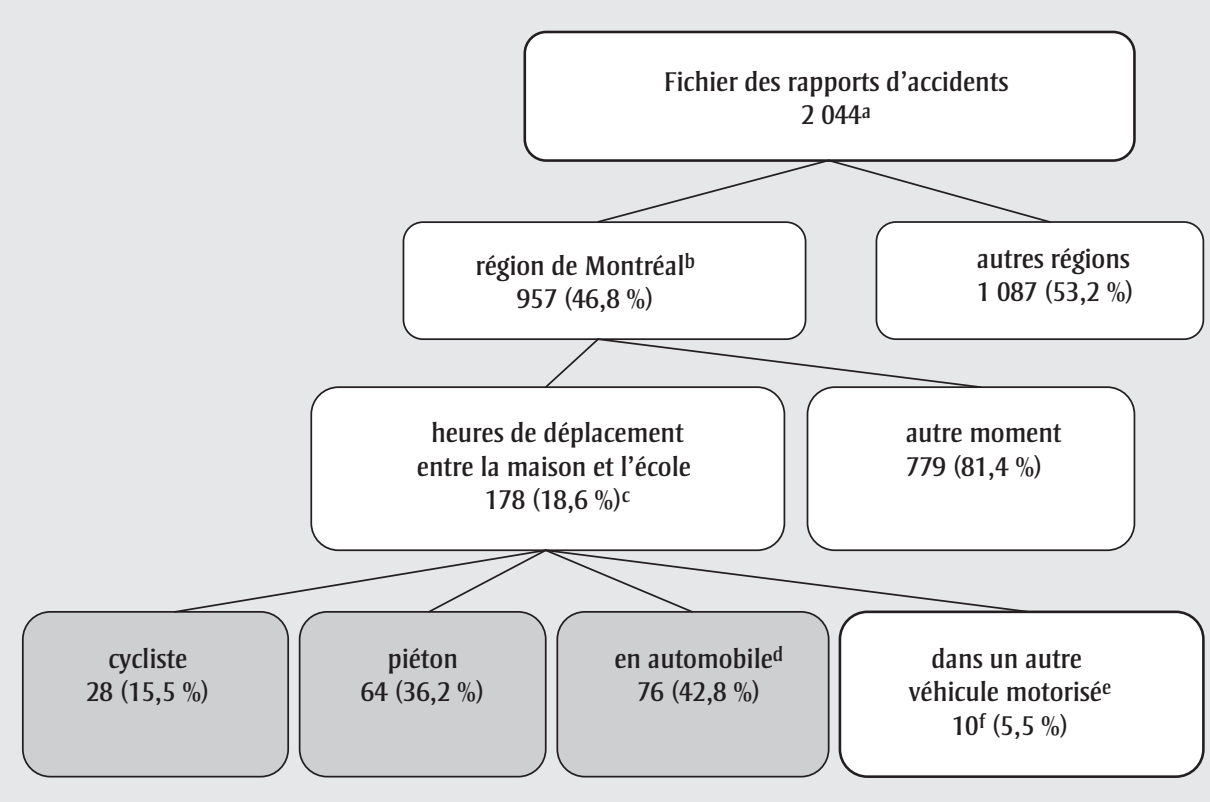

${ }^{a}$ Nombre annuel moyen d'enfants de 5 à 12 ans blessés comme piétons, cyclistes ou occupants d'un véhicule motorisé lors d'une collision impliquant un véhicule routier sur la voie publique, Québec, 2003-2007.

${ }^{\mathrm{b}}$ Répartition des blessés dans la région de Montréal (territoire couvert par l’Enquête origine-destination de 2003) grâce au code municipal.

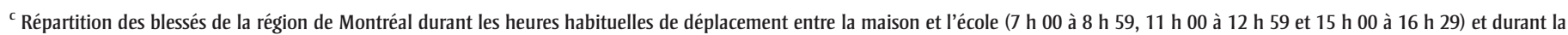
période scolaire (200 jours).

d Enfants blessés comme occupant d'une automobile, d'une camionnette ou d'un véhicule utilitaire sport.

e Enfants blessés comme occupant d'un véhicule motorisé d'un autre type (autobus scolaire, camion lourd, etc.).

${ }^{f}$ Parmi ces 10 blessés, 6 l'ont été comme occupants d'un autobus scolaire.

\section{Parts modales et kilométrage parcouru}

En 2003, 12799 enfants âgés de 5 à 12 ans ont participé à l'Enquête origine-destination dans la région de Montréal (tableau 1). Globalement, ces enfants (l'échantillon) sont associés à un total de 22819 déplacements entre la maison et l'école durant une journée de semaine ordinaire, dont 29,6\% en automobile, $32,5 \%$ à pied et $1,4 \%$ à vélo (les autres déplacements sont effectués principalement par autobus scolaire). Quatre-vingt-dix-huit pour cent (98,1 \%) des déplacements à pied et 86,1 \% des déplacements à vélo sont faits à moins de 1,6 kilomètre de l'école, contre 57,5\% des déplacements en automobile. En tenant compte des facteurs de pondération, on estime qu'en 2003, 332700 enfants de 5 à 12 ans (la population) se sont déplacés entre la maison et l'école dans la région de Montréal et que, globalement, ces enfants ont été associés à 588800 déplacements, dont $29,2 \%$ en automobile, 33,8 \% à pied et $1,3 \%$ à vélo.

Le tableau 2 présente les données relatives à l'échantillon selon le mois de l'enquête : 53,9\% de l'ensemble des déplacements tous modes confondus ont été effectués en septembre et octobre et $46,1 \%$ en novembre et décembre, mais respectivement $94,2 \%$ et $5,8 \%$ pour les déplacements à vélo. Le tableau 3 présente le kilométrage parcouru en une année par la population étudiée pour chaque mode de déplacement (colonne « kilométrage ", en milliers de kilomètres).

\section{Risque de blessures}

Dans la région de Montréal, entre 2003 et 2007, le taux annuel moyen de blessures chez les enfants de 5 à 12 ans lors des heures habituelles de déplacement entre la maison et l'école a été de 69 blessés par 100 millions de kilomètres parcourus en automobile (incluant camionnette et véhicule utilitaire sport) contre 314 pour les déplacements à pied et à 1519 pour les déplacements à vélo (tableau 3). Durant cette période, le risque relatif de blessures a été plus important pour les déplacements à pied (4,6, IC à $95 \%: 4,3$ à 5,1$)$ et à vélo $(22,2$, IC à $95 \%: 14,3$ à 30,0$)$ que pour les déplacements en automobile (groupe de référence). De plus, le risque de blessures lié aux déplacements à vélo était significativement plus élevé que le risque de blessures lié aux déplacements à pied. Les mêmes résultats ont été observés chez les enfants de 5 à 8 ans et chez ceux de 9 à 12 ans.

\section{Impact d'un transfert modal}

Un transfert modal de $10 \%$ du kilométrage parcouru en automobile vers les déplacements à pied chez les jeunes demeurant à moins de 1,6km de l'école 
TABLEAU 1

Description de l'échantillon et de la population étudiée selon les modes de déplacement

\begin{tabular}{|c|c|c|c|c|c|c|c|}
\hline \multirow[t]{3}{*}{ Modes de déplacement } & \multicolumn{4}{|c|}{ Échantillon ${ }^{a}$} & \multicolumn{3}{|c|}{ Population' (estimé) } \\
\hline & \multirow{2}{*}{$\begin{array}{l}\text { Nombre } \\
\text { d'enfants }\end{array}$} & \multicolumn{3}{|c|}{ Déplacements } & \multirow{2}{*}{$\begin{array}{c}\text { Nombre } \\
\text { d'enfants }^{c}\end{array}$} & \multicolumn{2}{|c|}{ Déplacements $^{d}$} \\
\hline & & $\mathbf{n}$ & $\%$ & $\begin{array}{l}\text { de moins de } \\
1,6 \mathrm{~km}^{\mathrm{e}}(\%)\end{array}$ & & $\mathbf{n}$ & $\%$ \\
\hline Auto $^{f}$ & 4661 & 6752 & 29,6 & 57,5 & 119700 & 172000 & 29,2 \\
\hline Marche & 3867 & 7413 & 32,5 & 98,1 & 104900 & 199000 & 33,8 \\
\hline Vélo & 156 & 325 & 1,4 & 86,1 & 3700 & 7500 & 1,3 \\
\hline Autres (autobus scol.) & $5176(4637)$ & 8329 (7 114) & $36,5(31,2)$ & $40,8(42,3)$ & 131400 (113 400) & 210000 (184500) & $35,7(31,3)$ \\
\hline Total $^{8}$ & 12799 & 22819 & 100,0 & 65,6 & 332700 & 588800 & 100,0 \\
\hline
\end{tabular}

Source : Enquête origine-destination menée dans la région de Montréal (2003) ${ }^{12}$.

${ }^{a}$ Enfants âgés de 5 à 12 ans ayant participé à l'Enquête origine-destination et déplacements effectués par ces enfants en une journée entre la maison et l'école.

b Nombre d'élèves du primaire âgés de 5 à 12 ans de la région de Montréal et nombre de déplacements effectués par ces enfants en une journée entre la maison et l'école.

c Après application des facteurs de pondération pour les personnes.

${ }^{\mathrm{d}}$ Après application des facteurs de pondération pour les déplacements.

e Déplacements entre la maison et l'école de moins de 1,6 km.

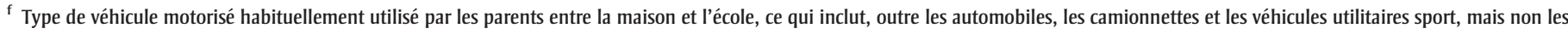
autobus, les camions lourds, les véhicules commerciaux et les véhicules tout-terrain.

${ }^{g}$ Les totaux pour le nombre d'enfants ne correspondent pas à la somme des nombres d'enfants selon le mode de déplacement car un même enfant peut utiliser plus d'un mode de déplacement par jour.

aurait occasionné 1,8 blessés de plus par année dans la région de Montréal, ce qui aurait représenté une augmentation de $1,1 \%(1,8 / 168,2)$ du nombre annuel moyen d'enfants blessés dans cette région lors des déplacements entre la maison et l'école à pied, à vélo et en automobile en 2003-2007 (tableau 4). Cette augmentation aurait été respectivement de $2,2 \%, 3,3 \%, 4,3 \%$ et $5,5 \%$ pour des transferts de $20 \%, 30 \%$, $40 \%$ et $50 \%$ (figure 2). Par ailleurs, avec un transfert de $10 \%$ du kilométrage parcouru en automobile vers les déplacements à vélo, il y aurait eu 12,2 blessés de plus par année, ce qui aurait représenté une augmentation de 7,3 \% (12,2/168,2) du nombre annuel moyen d'enfants blessés dans la région de Montréal lors des heures habituelles de déplacements entre la maison et l'école pour 2003-2007. Cette augmentation aurait été respectivement de 14,5\%, $21,7 \%, 29,0 \%$ et $36,2 \%$ pour des transferts de $20 \%, 30 \%$, $40 \%$ et $50 \%$ (figure 2).

\section{Analyse}

Dans la région de Montréal, entre 2003 et 2007, en moyenne 168 enfants âgés de 5 à 12 ans ont été blessés chaque année, 64

TABLEAU 2

Répartition de l'échantillon selon le mois de l'enquête, tous modes de déplacement confondus et déplacements à vélo, région de Montréal, 2003

\begin{tabular}{|c|c|c|c|c|c|c|}
\hline \multirow[t]{3}{*}{ Mois de l'enquête } & \multirow{2}{*}{\multicolumn{2}{|c|}{$\begin{array}{c}\text { Enfants }^{\mathrm{a}} \\
\text { Tous modes }\end{array}$}} & \multicolumn{4}{|c|}{ Déplacements ${ }^{b}$} \\
\hline & & & \multicolumn{2}{|c|}{ Tous modes } & \multicolumn{2}{|c|}{ Vélo } \\
\hline & $\mathbf{n}$ & $\%$ & $\mathbf{n}$ & $\%$ & $\mathbf{n}$ & $\%$ \\
\hline Septembre & 2727 & 21,3 & 5017 & 22,0 & 155 & 47,7 \\
\hline Octobre & 4087 & 31,9 & 7277 & 31,9 & 151 & 46,5 \\
\hline Novembre & 3332 & 26,0 & 5975 & 26,2 & 17 & 5,2 \\
\hline Décembre & 2653 & 20,7 & 4550 & 19,9 & 2 & 0,6 \\
\hline Total & 12799 & 100,0 & 22819 & 100,0 & 325 & 100,0 \\
\hline
\end{tabular}

Source : Enquête origine-destination menée dans la région de Montréal (2003) ${ }^{12}$.

a Enfants âgés de 5 à 12 ans ayant participé à l'Enquête origine-destination.

b Déplacements effectués en une journée entre la maison et l'école par les enfants âgés de 5 à 12 ans ayant participé à l'Enquête origine-destination. comme piétons, 28 comme cyclistes et 76 comme occupants d'une automobile, lors des heures habituelles de déplacement entre la maison et l'école, durant la période scolaire, ce qui représente presque un blessé par journée scolaire. Dans plus de $90 \%$ des cas, il s'agissait de blessures légères.

Le taux annuel moyen de blessures par 100 millions de kilomètres parcourus a été de 69 blessés pour les occupants d'une automobile, 314 blessés pour les piétons et 1519 blessés pour les cyclistes. Ces résultats suggèrent que les enfants qui se déplacent à pied ou à vélo courent davantage de risques de blessures (risques relatifs respectifs de 4,6 et 22,2) que ceux se déplaçant en automobile (référence), et que les déplacements à vélo induisent un plus grand risque de blessures que les déplacements à pied. Les mêmes tendances ont été observées pour les deux groupes d'âge étudiés. Les résultats d'analyses complémentaires réalisées dans les régions de Québec et Trois-Rivières font également état des mêmes tendances (données disponibles sur demande). Des tendances similaires ont été observées lors d'une étude menée à l'échelle des ÉtatsUnis $^{8}$ pour la période 1991-1999. Dans cette étude, le risque relatif de blessures par rapport aux déplacements en auto- 
TABLEAU 3

Estimation du risque de blessures chez les enfants de 5 à 12 ans lors des déplacements entre la maison et l'école, selon l'âge et le mode de déplacement, région de Montréal, 2003-2007

\begin{tabular}{|c|c|c|c|c|c|c|c|}
\hline Âge (ans) & $\begin{array}{c}\text { Modes de } \\
\text { déplacement }\end{array}$ & $\begin{array}{l}\text { Blessés (nombre } \\
\text { moyen par an) }\end{array}$ & $\begin{array}{c}\text { Kilométrage } \\
\text { (en milliers de } \mathrm{km} \text { ) }\end{array}$ & $\begin{array}{c}\text { Taux (pour } \\
100 \text { millions de } \mathbf{k m} \text { ) }\end{array}$ & IC à 95 \% & $\mathbf{R R}$ & IC à $95 \%$ \\
\hline \multirow[t]{2}{*}{5 à 8} & Automobile & 36,8 & 56705 & 65 & $(60-71)$ & 1 (réf.) & - \\
\hline & Vélo & 6,6 & 294 & 2244 & (1451-4957) & 34,6 & $(15,4-53,8)$ \\
\hline \multirow[t]{2}{*}{9 à 12} & Automobile & 39,4 & 54409 & 72 & (67-79) & 1 (réf.) & - \\
\hline & Marche & 40,4 & 12028 & 311 & $(294-330)$ & 4,3 & $(3,8-4,7)$ \\
\hline \multirow[t]{3}{*}{5 à 12} & Automobile & 76,2 & 111114 & 69 & $(64-73)$ & 1 (réf.) & - \\
\hline & Marche & 64,4 & 18634 & 314 & $(300-330)$ & 4,6 & $(4,3-5,1)$ \\
\hline & Vélo & 27,6 & 1817 & 1519 & (1129-2319) & 22,2 & $(14,3-30,0)$ \\
\hline
\end{tabular}

Abréviations : IC, intervalle de confiance; réf., référence; RR, risque relatif.

mobile chez les enfants de 5 à 10 ans était 9,4 fois plus élevé pour les déplacements à pied et 34 fois plus élevé pour les déplacements à vélo (ces risques relatifs ont été calculés par rapport à des taux de blessés par 100 millions de kilomètres parcourus). Les mêmes tendances ont également été observées dans une étude menée en Nouvelle-Zélande ${ }^{9}$ portant sur la période 2003-2005, dans laquelle le risque relatif de blessures par rapport aux déplacements en automobile chez les enfants de 5 à 17 ans était 2,2 fois plus élevé pour les déplacements à pied et 14,6 fois plus élevé pour les déplacements à vélo (ces risques relatifs ont été calculés par rapport à des taux de blessés par million d'heures de parcours). À notre connaissance, ces deux études sont les seules concernant l'évaluation du risque de blessures associé aux déplacements des élèves du primaire entre la maison et l'école, durant la période scolaire et en contrôlant pour l'exposition (kilométrage parcouru ou heures de parcours).

L'effet d'un transfert modal sur le nombre de blessés a été évalué pour cinq scénarios de transfert des déplacements par automobile vers les déplacements à pied ou à vélo, allant de $10 \%$ à $50 \%$. Un transfert de $20 \%$ des déplacements par automobile pour les enfants demeurant à moins de 1,6 km de l'école vers la marche aurait occasionné 3,7 blessés de plus par année, ce qui aurait représenté une augmentation de $2,2 \%$ du nombre annuel moyen d'enfants blessés dans cette région lors des heures habituelles de déplacements entre la maison et l'école, et 24,4 blessés de plus et une augmentation de 14,5\% dans le cas d'un transfert vers le vélo. À notre connaissance, ce type d'évaluation n'a fait l'objet d'aucune publication scientifique, ce qui empêche toute comparaison des résultats.

\section{Forces et limites de l'étude}

Ces analyses ont été faites en considérant tous les enfants de 5 à 12 ans inclus dans le Fichier des rapports d'accidents de véhicules routiers durant la période 2003 à 2007 (numérateur) et tous ceux ayant participé à l'Enquête origine-destination menée en 2003 dans la région de Montréal (dénominateur). Le taux de blessures a été évalué en contrôlant pour le nombre de kilomètres parcourus (dénominateur), ce qui permet de comparer le nombre de

TABLEAU 4

Effet de cinq scénarios de transfert du kilométrage parcouru en automobile entre la maison et l'école vers les déplacements à pied ou à vélo sur le nombre annuel moyen d'enfants de 5 à 12 ans blessés, région de Montréal, 2003-2007

\begin{tabular}{|c|c|c|c|c|c|c|c|c|}
\hline \multirow{2}{*}{\multicolumn{2}{|c|}{ Scénarios de transfert du kilométrage parcouru ${ }^{\mathrm{a}}$}} & \multirow{2}{*}{\multicolumn{3}{|c|}{$\begin{array}{l}\text { Estimation du nombre annuel moyen de blessés } \\
\text { lié à chaque scénario de transfert, selon le mode } \\
\text { de déplacement }{ }^{\text {b }}\end{array}$}} & \multicolumn{4}{|c|}{$\begin{array}{c}\text { Effet du transfert sur le nombre annuel moyen de } \\
\text { blessés dans la région }{ }^{c}\end{array}$} \\
\hline & & & & & \multicolumn{2}{|c|}{ Auto vers marche } & \multicolumn{2}{|c|}{ Auto vers vélo } \\
\hline Proportion de transfert & Nombre de km & en automobile & à pied & en vélo & Nombre & $(\%)$ & Nombre & $\%$ \\
\hline $10 \%$ & 881540 & 0,60 & 2,44 & 12,79 & $+1,8$ & $(+1,1)$ & $+12,2$ & $(+7,3)$ \\
\hline $20 \%$ & 1763080 & 1,21 & 4,88 & 25,57 & $+3,7$ & $(+2,2)$ & $+24,4$ & $(+14,5)$ \\
\hline $30 \%$ & 2644620 & 1,81 & 7,33 & 38,36 & $+5,5$ & $(+3,3)$ & $+36,5$ & $(+21,7)$ \\
\hline $50 \%$ & 4407700 & 3,03 & 12,21 & 63,93 & $+9,2$ & $(+5,5)$ & $+60,9$ & $(+36,2)$ \\
\hline
\end{tabular}

a Les scénarios de transfert du kilométrage parcouru portent sur les enfants habitant à moins de 1,6 km de l'école.

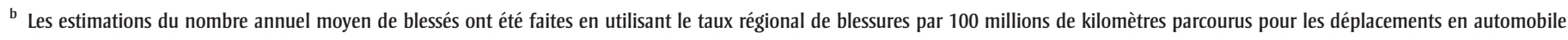
(69), à pied (314) et à vélo (1 519), voir tableau 3.

c L'effet du transfert modal est calculé sur l'ensemble des enfants blessés dans la région de Montréal. 
FIGURE 2

Effet de cinq scénarios de transfert du kilométrage parcouru en automobile par les enfants résidant à moins de 1,6 km de l'école vers la marche et le vélo sur le nombre annuel moyen d'enfants de 5 à 12 ans blessés lors des heures habituelles de déplacement entre la maison et l'école (en \%), région de Montréal, 2003-2007

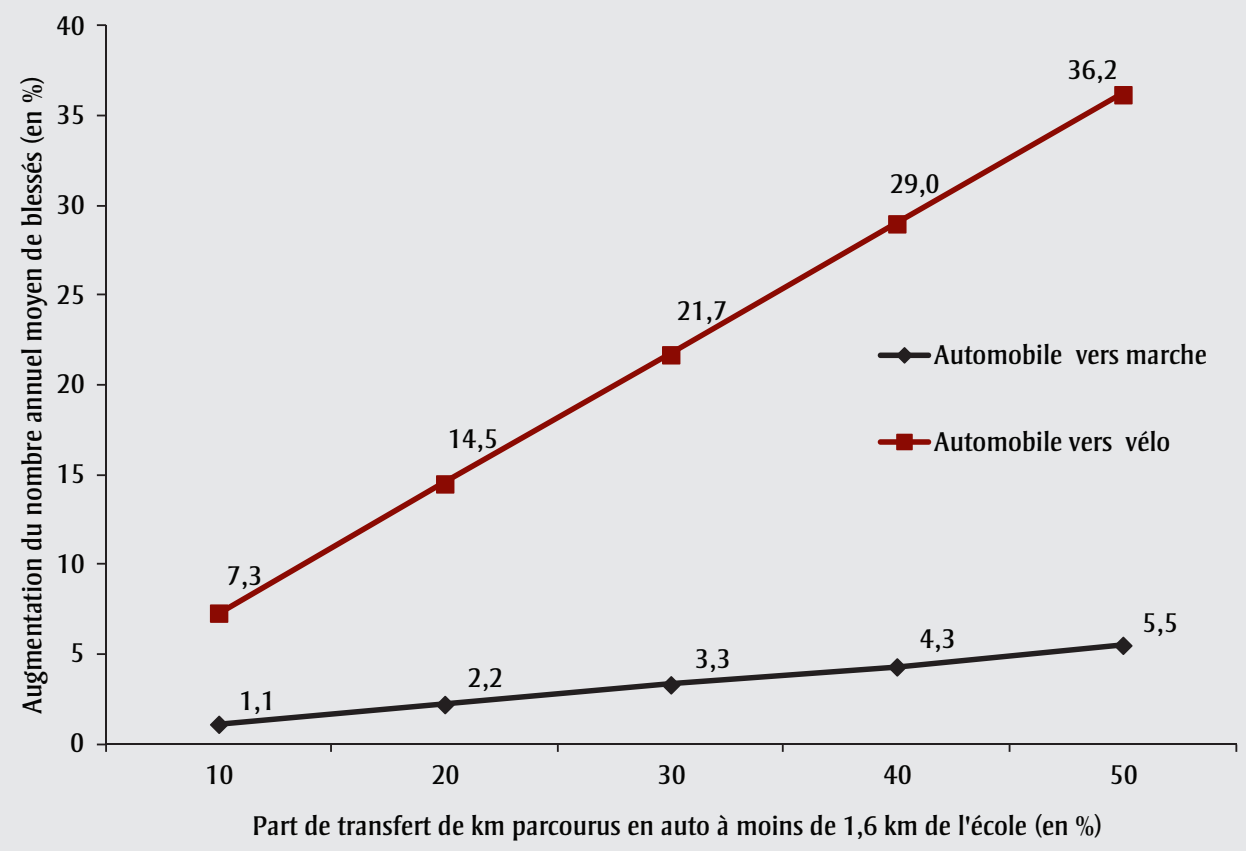

blessés en tant que piétons, cyclistes ou occupants d'une automobile pour une même distance parcourue. Le nombre total de kilomètres parcourus durant la période scolaire a été estimé en multipliant la distance parcourue en une journée par le nombre de journées d'école (200 jours). Or le moment de la collecte des données durant l'année est susceptible d'influencer le mode de déplacement choisi. On sait à ce sujet que l'enquête a touché la moitié des enfants en septembre et octobre et la moitié en novembre et décembre. On peut supposer que les entrevues menées en septembre et octobre nous renseignent sur l'exposition des enfants durant les mois de l'année scolaire où la température est plutôt clémente, et donc plus propice aux déplacements à pied ou à vélo (septembre, octobre, avril, mai et juin), alors que les entrevues menées en novembre et décembre renseignent sur l'exposition des enfants durant les mois où la température est moins clémente (novembre, décembre, janvier, février et mars). D’ailleurs, la répartition des déplacements selon le mois de l'enquête en témoigne : la plupart des déplacements à vélo sont effectués en septembre et octobre contre seulement quelques-uns en novembre et décembre. Fait à noter, bien que cette estimation du kilométrage parcouru se rapporte à l'exposition des enfants durant l'année de l'enquête (2003), elle est sans doute aussi un bon indicateur de l'exposition des enfants pour l'ensemble de la période étudiée (2003 à 2007), car il serait surprenant que la part modale des déplacements à pied, à vélo et par automobile ait changé significativement durant cette période. Par ailleurs, le fait d'estimer le kilométrage parcouru en fonction de la distance à vol d'oiseau (longueur d'une ligne droite tracée entre la maison et l'école) constitue probablement l'une des limites de cette étude : cette distance est généralement plus courte que celle réellement parcourue par les enfants. Une autre limite de l'étude concerne la non exhaustivité des données comprises dans le Fichier des rapports d'accidents pour les blessures causées lors d'une collision impliquant un véhicule motorisé, sans compter le fait que ce fichier n'inclut pas les blessures n'impliquant pas de véhicule motorisé ${ }^{14-17}$.

L'impact d'un transfert modal des déplacements en automobile vers les déplace- ments actifs (marche et vélo) sur le nombre d'enfants blessés a été évalué auprès des enfants habitant à moins de 1,6 km de leur école. Cette évaluation a été faite en utilisant les taux de blessures calculés à l'échelle de la région de Montréal (taux régional), car les données disponibles dans le Fichier des rapports d'accidents de véhicules routiers ne permettent pas de répartir le numérateur (nombre de blessés) selon la distance réelle à l'école (impossibilité de calculer le taux spécifique de blessures des enfants demeurant à moins de 1,6 km d'une école donnée). Pour cette raison, on doit faire le postulat que le taux de blessures calculé à l'échelle de la région est similaire au taux de blessures des enfants habitant à moins de 1,6 km de leur école. Ce postulat est assez plausible pour les piétons et les cyclistes : 98,1 \% des déplacements à pied et $86,1 \%$ de ceux à vélo dans la région de Montréal sont faits à moins de 1,6 km de l'école. Cela est plus difficile à évaluer toutefois pour les enfants se déplaçant en automobile, puisque la part de ces déplacements faite à l'intérieur de ce périmètre est moins importante $(57,5 \%)$. De plus, le fait d'utiliser le taux régional (qui est en 
fait un taux moyen) permet d'évaluer l'impact d'un transfert modal uniquement à l'échelle de la région (effet moyen), alors que cet effet peut varier d'un quartier à l'autre en raison d'une variation du risque de blessures (variation spatiale). Finalement, il importe de préciser que l'impact d'un transfert modal a été évalué sans tenir compte du fait que le risque de blessures pour les piétons et les cyclistes pourrait diminuer en raison de la diminution du nombre de véhicules en circulation. Mais l'analyse des données disponibles laisse croire que cet effet serait marginal : un transfert de $20 \%$ des déplacements en automobile pour les enfants demeurant à moins de 1,6 km de l'école serait associé à une réduction de 13780 automobiles (20 \% x 68900 enfants), ce qui représente un très faible pourcentage du nombre total de véhicules motorisés dans la région.

\section{Conclusion}

Très peu d'études ont évalué le risque de blessures chez les élèves du primaire lors des déplacements entre la maison et l'école durant la période scolaire, et cette étude est la première du genre au Québec. De plus, pour autant que nous le sachions, c'est la première fois qu'une étude évalue l'impact auprès de cette clientèle d'un transfert modal des déplacements en automobile vers les déplacements actifs (marche et vélo) sur le nombre de blessés.

Globalement, les résultats observés suggèrent que les programmes de promotion du transport actif auprès des élèves du primaire dans la région de Montréal pourraient, en provoquant un transfert des déplacements en automobile vers les déplacements à pied ou à vélo, avoir pour effet d'augmenter le nombre d'enfants blessés (blessures légères pour la plupart) lors des déplacements entre la maison et l'école, si aucune mesure n'est prise pour favoriser davantage la sécurité des piétons et des cyclistes. Ce type de programme provoque habituellement un transfert de $20 \%$ ou moins des déplacements en automobile auprès des jeunes demeurant à une distance de l'école propice à la marche ou au vélo ${ }^{3}$. L'impact d'un tel transfert serait plus important dans le cas d'un transfert complet vers les déplace- ments à vélo, mais ce scénario est peu probable étant donné que ce mode de déplacement est beaucoup moins populaire que la marche.

Plusieurs mesures sont disponibles pour sécuriser les déplacements à pied ou à vélo entre la maison et l'école ${ }^{18}$ : aménagement de l'environnement routier (ex. : dos-d'âne allongé, réduction de la largeur des rues, avancées de trottoir, feux de piétons), brigadier scolaire, accompagnement des enfants par un adulte, port d'un casque de vélo et cours d'éducation à la sécurité routière. La priorité doit toutefois être accordée aux mesures visant à sécuriser l'environnement routier, parce qu'elles se sont révélées efficaces ou prometteuses ${ }^{19}$ et parce que, une fois implantées, leur effet protecteur est toujours présent, quel que soit l'âge, le sexe ou le milieu socioéconomique de l'enfant. Ces mesures ont le potentiel de contrer l'impact du transfert modal découlant d'un programme de promotion du transport actif au primaire, étant donné qu'elles protègent non seulement les nouveaux piétons et cyclistes, mais également les enfants qui se déplaçaient à pied ou à vélo avant l'implantation du programme, et ils demeurent les plus nombreux. L'inclusion de ce type de mesures dans ces programmes est importante à la fois pour assurer la sécurité des enfants et pour promouvoir le transport actif auprès de cette clientèle, l'insécurité routière étant, comme nous l'avons mentionné, l'une des principales raisons invoquées par les parents pour privilégier l'automobile aux dépens de modes de déplacement actifs.

\section{Références}

1. Osborne P. Safe routes for children : what they want and what works. Children, youth and environments. 2005;15(1):234-239.

2. Vélo Québec. Mon école à pied, à vélo, mode d'emploi. Montréal (Qc) : Vélo Québec; 2008.

3. Chillon P, Eveson KR, Vaughn A, Ward DS. A systematic review of interventions for promoting active transportation to school. International Journal of Behavioral Nutrition and Physical Activity. 2011;8:10.
4. Schieber RA, Thompson NJ. Developmental risk factors for childhood pedestrian injuries. Injury Prevention. 1996;2:228-236.

5. Cloutier MS. Connaissance, croyance et représentation du risque routier piéton chez les parents d'enfants du primaire : implication pour les politiques de promotion des transports actifs. DIRE. 2008;automne:21-26.

6. Lewis P et collab. Le transport actif et le système scolaire à Montréal et à Trois Rivières. Analyse du système d'acteurs concernés par le transport actif des élèves des écoles primaires au Québec. Montréal (Qc) : Groupe de recherche Ville et Mobilité; 2008.

7. Centers for Disease Control and Prevention. Barriers to children walking to or from school-United States. MMWR Morb Mortal Wkly Rep. 2005;54:949-952.

8. Transportation research Board. The relative risks of school travel : A national perspective and guidance for local community risk assessment. Washington, DC : Transportation research Board; 2002. (special report 269).

9. Schofield GM, Gionotti S, Badland HM, Hinckson EA. The incidence of injuries traveling to and from school by travel mode. Preventive Medicine. 2008;46(1):74-76.

10. Agence Métropolitaine du Transport. Enquête Origine-Destination 2003 : la mobilité des personnes dans la région de Montréal. Montréal (Qc) : Agence Métropolitaine du Transport; 2005.

11. Société de l'assurance automobile du Québec (SAAQ). Jeunes victimes âgées entre 5 et 12 ans blessées en période scolaire ou hors période scolaire : 2003-2007 (compilation spéciale). Québec (Qc) : SAAQ; 2008.

12. Ministères des transports du Québec (MTQ). Part modale des déplacements effectués par les enfants âgés de 5 à 12 ans, entre la maison et l'école, durant la période scolaire : régions de Montréal (1996 et 2003), Québec (1991 et 2006), Sherbrooke (1992 et 2003) et Trois-Rivières (2000). Québec (Qc) : MTQ, Service de la modélisation des systèmes de transport; 2009.

13. Research Triangle Institute. SUDAAN Language Manual, Volumes 1 and 2, Release 11. Research Triangle Park, NC : Research Triangle Institute; 2012. 
14. Kopjar B, Wickizer TM. Cycling to school-a significant health risk?. Injury Prevention. 1995;1:238-241.

15. Agence de la santé publique du Canada. Système canadien hospitalier d'information et de recherche en prévention des traumatismes : blessures associées aux bicyclettes (2006, 1 an et plus). Ottawa (Ont.) : Agence de la santé publique du Canada, Division de la surveillance de la santé et de l'épidémiologie; 2008.

16. Agran PF, Castillo DN, Winn DG. Limitations of data compiled from police reports on pediatric pedestrian and bicycle motor vehicle events. Accid Anal Prev. 1990; 22:361-370.

17. Dhillon PK, Lightstone AS, Peek-Asa C, Kraus JF. Assessment of hospital and police ascertainment of automobile versus childhood pedestrian and bicyclist collisions. Accid Anal Prev. 2001;33:529-537.

18. Burigusa G, Lavoie M, Maurice P, Hamel D, Duranceau A. Sécurité des élèves du primaire lors des déplacements à pied et à vélo entre la maison et l'école au Québec. Québec (Qc) : Institut national de santé publique du Québec; 2011. PDF (2 Mo) téléchargeable à partir du lien : http:// www.inspq.qc.ca/pdf/publications/1243 SecuriteElevesDeplacementsMaisonEcole .pdf

19. Retting RA, Ferguson SA, McCartt AT. A review of evidence-based traffic engineering measures designed to reduce pedestrian-motor vehicle crashes. Am J Public Health. 2003;93:1456-1463. 